\title{
A gestão da informação como proposta de inclusão em uma biblioteca especializada'
}

\author{
Information management as a proposal \\ for inclusion in a specialist library
}

Barbara Coelho NEVES²

Maria Célia Nery PADILHA ${ }^{3}$

\section{Resumo}

Esta comunicação apresenta o relato de experiência da implementação do Núcleo de Gestão da Informação e Inteligência administrado pela Biblioteca da Defensoria Pública do Estado da Bahia. O Núcleo tem o propósito de corroborar com o estoque informacional, baseando-se na captação do conhecimento tácito dos defensores públicos da Defensoria Pública do Estado da Bahia. Ambiciona-se a inclusão social e a preservação da memória dos direitos humanos empregada nas atividades defensoriais desenvolvidas. O maior benefício da criação do Núcleo de Gestão da Informação e Inteligência é proporcionar a disponibilidade de informação adquirida pelos defensores, de maneira estruturada, a todas as pessoas interessadas nos temas defensoriais apreendidos através de vivências jurídicas, congressos, cursos de capacitação, seminários, workshops, dentre outros. O Núcleo de Gestão da Informação e Inteligência da Biblioteca da Defensoria Pública do Estado da Bahia constitui importante meio para conectar eficientemente "aqueles que sabem" àqueles que "necessitam saber" e converter conhecimento pessoal em memória da organização. Para tanto, a proposta desenvolve a coleta, o armazenamento, gerenciamento e disseminação do conhecimento com uma metodologia baseada em modelos de gestão da informação e gestão do conhecimento, com o auxílio de adolescentes "menores aprendizes" em cumprimento de medida socioeducativa da Fundação Cidade Mãe.

Palavras-chave: Biblioteca especializada. Gestão da informação. Gestão do conhecimento. Inclusão social.

\begin{abstract}
This paperpresents the account of experiences with the implementation of the Information and Intelligence Management Center administered by the Library of the Public Defender's Office in the Brazilian state of Bahia. The aim of this Center is to corroborate the information inventory, based on the capture of tacit knowledge from the attorneys of the Public Defender's Office in Bahia. It aspires to social inclusion and the preservation of the memory of human rights as used in activities undertaken by these public defenders. The biggest benefit to be gained by creating the Center is to provide the availability of information gained by the public defenders in a structured form, to all persons interested in the topics of defense acquired through legal experiences, conferences, training courses, seminars, workshops, amongst others. The Information and Intelligence Management Center of the Library of the Public Defender's Office in Bahia is an important means to efficiently connect "those who know" with those who "need to know" and convert individual knowledge into organizational memory. To this end, the proposal builds on the collection, storage, management and dissemination of knowledge using a methodology based on information and knowledge management models, with the help of teenage "apprentices" in compliance with the socio-educational measures of the Fundação Cidade Mãe (literally the Mother-City Foundation).
\end{abstract}

Keywords: Specialized library. Information management. Knowledge management. Social inclusion.

\footnotetext{
1 Artigo elaborado a partir do Projeto Gestão da Informação e Inteligência da Escola Superior de Defensoria Pública do Estado da Bahia, ganhador do prêmio Ideias e Ações Inovadoras Governo do Estado da Bahia em 2010.

2 Doutoranda em Educação, Universidade Federal da Bahia, Programa de Doutorado em Educação. Reitor Miguel Calmon, s/n., Vale do Canela, 40210-340, Salvador, BA, Brasil. Correspondência para/Correspondence to: B.C. NEVES. E-mail: <barbaracoelho2000@yahoo.com.br>.

3 Defensora Pública Geral do Estado da Bahia. Salvador, BA, Brasil.

Recebido em 18/7/2011, reapresentado em 28/10/2011 e aceito para publicação 9/11/2011
} 


\section{Introdução}

O momento contemporâneo tem sido caracterizado pela rapidez da disseminação das informações, proporcionada pelas Tecnologias de Informação e Comunicação (TIC), consolidando a então chamada sociedade da informação. Esse é um fenômeno comum a todos os eixos - de ordem econômica, social, política e cultural -, o que leva a perceber que as transformações oriundas desse contexto afetam de maneira profunda pessoas e organizações.

Esta comunicação apresenta a implementação de um projeto de gestão da informação no âmbito organizacional, em especial em uma instituição de operacionalidade estatal, mantida pelo poder público. Consiste na síntese de uma proposta de implementação do Núcleo de Gestão da Informação e Inteligência para a Escola Superior da Defensoria Pública do Estado da Bahia (ESDEP), integrante dos Órgãos Auxiliares da Defensoria Pública do Estado da Bahia (DPE).

A Escola Superior da Defensoria Pública desenvolve um trabalho que contribui com a difusão da informação por meio do desenvolvimento de atividades que têm como finalidade promover o aperfeiçoamento profissional e cultural do quadro de pessoal da DPE. A ESDEP confere cursos de atualização, atividades de qualificação funcional, além de estimular o intercâmbio com organizações oficiais e entidades da sociedade civil. Sua missão compreende, como um de seus principais aspectos, o estímulo à preparação da carreira de seu quadro profissional, visando à disposição de informações atualizadas para o desenvolvimento profissional e institucional. Somente entre os anos de 2009 e 2010, a ESDEP estimulou a em mais de 30 eventos em âmbito nacional e internacional. Esses beneficiários participam de palestras, cursos, congressos, workshops, dentre outros, criando uma rede de relacionamentos (network) e de contextos de apreensão de conteúdos informacionais sobre as áreas defensoriais e afins.

Silva e Ribeiro (2008, p.21) vão dizer que "antes e depois do documento há a informação". Ou seja, a informação reina absoluta na sociedade, desde os tempos mais remotos. Diante disso, percebemos, antes de entrar- mos na ceara da gestão da informação, a necessidade de alinhar conceitualmente o que estamos compreendendo por informação. Acredita-se que a aproximação com o conceito de informação deu apoio ao desenho do projeto que ora se apresenta neste relato e tentou evitar o "[...] pântano do empirismo tecnicista imposto pela perigosa rotina do trabalho cotidiano [...]" (Silva; Ribeiro, 2008, p.22, grifo dos autores). Procurou-se também evitar esse pântano tecnicista proveniente da execução diária dos projetos, que insiste por aparecer nos relatos descritivos de implementação.

Desse modo, utilizou-se como ponto de partida o primeiro momento de compreensão de Silva e Ribeiro (2008, p.23), como "[...] a informação entre o sujeito individual que conhece, pensa, se emociona e interage com o mundo sensível à sua volta e a comunidade de sujeitos que comunicam entre si". Concorda-se com os autores acima de que é preciso adicionar à tal ilação elementos sociais. O contexto que deu origem a este texto envolve aspectos da estrutura organizacional, social e do registro documental. Dessa forma, a base que fundamenta o projeto encontra fôlego na definição de informação, que a entende como um "[...] conjunto estruturado de representações mentais codificadas (símbolos significantes) socialmente contextualizadas e passíveis de serem registradas num qualquer suporte material (papel, filme, banda magnética, disco compacto etc.) e, portanto, comunicadas de forma assíncrona e multidirecionada" (Silva; Ribeiro, 2008, p.37, grifo dos autores).

Com essa definição de informação, consegue-se abarcar todos os elementos que envolvem o projeto e a noção semântica empregada na expressão gestão da informação de que se vale este texto.

Os termos gestão da informação e inteligência são abordados sob a ótica de pesquisadores que os tratam pelo o viés da gestão da informação. Será usado o entendimento de Valentim (2002) sobre a expressão gestão da informação: informação trata-se de dados que sofreram algum tipo de análise sob o clivo de alguma área ou atribuição de significado e em sintonia com o público a que se destina. O termo inteligência, na perspectiva que interessa nesta comunicação, corresponde ao conceito de inteligência competitiva que, de acordo com Valentim 
(2002), é um processo contínuo. Sua maior complexidade está no fato de estabelecer relações e conexões de forma a gerar inteligência para a organização, na medida em que cria estratégias para cenários futuros, possibilitando tomadas de decisão de maneira mais segura e assertiva.

A gestão da informação implica a adoção de práticas gerenciais compatíveis com as conclusões citadas sobre os processos de criação e aprendizado individual e, também, a coordenação sistêmica de esforços em vários planos: organizacional; estratégico e operacional; normas formais e informais (Molina, 2008).

Segundo Valetim (2002), a gestão da informação pode ser definida como um processo que trabalha no âmbito do conhecimento explícito, ou seja, são dados e informações que já estão consolidados em algum tipo de veículo de comunicação. Como exemplo, pode-se citar desde o livro impresso até a rede Internet.

Atualmente, o volume de informações cresce a uma grande velocidade, fazendo com que haja certa dificuldade em organizá-las e disponibilizá-las. Existe a necessidade de se difundir rapidamente o maior volume possível de informações em cada área. A gestão da informação é um meio de coletar, organizar e disseminar esse conhecimento gerado.

De acordo com Oliveira e Bertucci (2003), a gestão da informação se tornou um instrumento estratégico utilizado, na maioria das vezes e principalmente, no âmbito institucional, para controlar e auxiliar decisões, por meio da fluidez do fluxo da informação, análise prévia e consolidação das informações para os usuários. Essa perspectiva é a que, geralmente, vem norteando as concepções de gestão da informação no contexto organizacional. A ela adicionaremos, portanto, elementos como a interação, possibilidade de colaboração e compartilhamento, visando à multiplicidade da informação no âmbito da instituição. Compreendemos que o comportamento informacional dos funcionários e executivos é alterado diante da demanda da informação e daqueles que lidam profissionalmente com ela, em um papel de mediação que absorve a característica de multiplicadores.

Analisando por essa ótica, fez-se necessária a implementação de um serviço de gestão da informação em que se possa desenvolver, de maneira estratégica, a coleta, o armazenamento e a disseminação do estoque de informações dos defensores públicos e demais beneficiários pelas atividades desenvolvidas pela ESDEP. Outra frente que esta comunicação também vislumbra está relacionada à inclusão social que o projeto visa a proporcionar, uma vez que, além de garantir que todos tenham acesso às informações, envolve o treinamento e a qualificação dos menores aprendizes da Fundação Cidade Mãe, em cumprimento de atividades socioeducativas na DPE. Como resultado, apresentamos aos pares algumas observações dessa experiência, que se acredita corroborar com o estoque informacional baseado na capitação do conhecimento tácito da DPE.

Assim, esta comunicação relata os principais pontos que fundamentaram o projeto de desenvolvimento do Núcleo de Gestão da Informação e Inteligência da Escola Superior da Defensoria Pública do Estado da Bahia, ambientado na Biblioteca da ESDEP, com o propósito de divulgar essa proposta de convergência entre gestão da informação e inclusão social. Contudo, não foi propósito desta comunicação exaurir todo o contexto do projeto em fase de implementação, como também abordar todos os constructos dos termos apresentados, mas sim trazer os conceitos que norteiam e a apreensão que se faz deles neste Projeto.

\section{Gestão da informação e inclusão social}

De acordo com Borges e Souza (2003), a informação é um dos insumos mais importantes para o desenvolvimento organizacional quando disponibilizada com rapidez e precisão, refletindo as demandas internas e externas (local e global). Essa necessidade de explorar ao máximo os serviços e produtos de informação no ambiente organizacional vem sendo um desafio para os profissionais de informação.

Para Moresi e Mendes (2010), quando essas mudanças não são planejadas na organização, a resistência interna aumenta, gerando obstáculos que vão da incerteza à falta de disposição, conduzindo a fraquezas e falhas.

Grandes empresas vêm se utilizando das linhas de ação desenhadas a partir dos temas gestão da informação e do conhecimento para se posicionar no mercado 
globalizado, com a perspectiva de se adiantarem aos fenômenos que tal contexto demanda. Essas organizações desenvolvem verdadeiras inteligências que se antecipam às demandas informacionais, que, nesse caso, podem ser, dentre outras, de origem financeira, mercadológica, logística e cultural. Borges e Souza (2003) aponta que além do viés mercadológico, torna-se necessário considerar as relações existentes entre a unidade de informação e a comunidade a que ela atende. Destarte, foi se aproximando de abordagens e relatos sobre inteligência e produtos informacionais que nos questionamos quanto ao que pode ser feito, com base na gestão da informação e do conhecimento, para fomentar a ideia de "inteligência" em uma instituição pública.

Entende-se que um núcleo de inteligência no âmbito de uma instituição, em que pese sua característica de órgão público, deve considerar os aspectos que recheiam a definição de informação mencionada na introdução: informação significativa, em contexto social, potencialmente registrável, podendo ser disseminada. De posse desses aspectos, o problema que nos parece subjacente é identificar possíveis meios de se converter conhecimento tácito em explícito. Nesse caso, identifica-se essa possibilidade observando que alguns defensores e funcionários tinham acesso às informações valiosas para as atividades internas de cunho coletivo dos setores específicos, onde estes estavam alocados.

A intenção é globalizar o conhecimento local que, citado por Moresi e Mendes (2010), tem um estímulo forte no quesito que prevê o nivelamento do conhecimento na instituição. Esse é um item capacitador de conhecimento que converge com a nossa proposta em fazer que o máximo quantitativo de usuários tenha acesso, em ambiente externo, às informações adquiridas nos cursos de qualificação do público interno da instituição.

Constitui uma das propostas principais da ESDEP a criação de um ambiente que vise à organização/ordenação e ao atendimento da demanda informacional intra e extrainstitucional concernente no acesso aos documentos impressos, digitais e virtuais sobre os temas de interesse da área jurídica e afins. O entendimento da associação entre gestão da informação e inclusão social garante a relevância e continuidade do projeto.
O maior benefício da criação do Núcleo de Gestão da Informação e Inteligência é proporcionar a disponibilidade da informação adquirida pelos beneficiários a todas as pessoas interessadas nos temas defensoriais. Trata-se de disseminar para todos os membros da DPE aspectos do conteúdo e principais ideias a que os beneficiados tiveram acesso através de congressos, cursos de capacitação, vivências jurídicas, seminários, workshops, dentre outros, constantemente viabilizados pela Instituição. Os ambientes que se propõem a gerir informação são importantes para conectar eficientemente "aqueles que sabem" com aqueles que "necessitam saber" e converter conhecimento pessoal em conhecimento da organização.

É desse modo que se justifica a criação do Núcleo de Gestão da Informação e Inteligência da ESDEP, respaldada no cenário atual, que envolve as instituições públicas, que vislumbram o aprender a aprender, e na possibilidade de tornar explícito e codificado os processos tácitos individuais e coletivos de construção de conhecimento no ambiente organizacional (Molina, 2008), ou seja, criar condições para que o conhecimento aflore e seja partilhado em função do interesse de todos e da missão da organização.

Com base no comentário de Stewart (1991), o conhecimento das organizações é a soma de tudo que as pessoas sabem e é essa inteligência que confere a vantagem competitiva. Contudo, também as instituições públicas acumulam conhecimentos na mente das pessoas e informações de forma não estruturadas. Apesar de diante da superestrutura (Estado) as organizações terem objetivos diferentes das instituições públicas, entende-se, concordando com Molina (2008), que o desenvolvimento de práticas de gestão da informação propicie a configuração de ambiente de aprendizado e compartilhamento. A gestão da informação trabalha com a perspectiva de coleta, validação, avaliação e aplicação.

O modelo de gestão do conhecimento de Nonaka e Takeuchi (1995) vem sendo bastante difundido em projetos de gestão da informação de grandes organizações brasileiras, a exemplo da Petrobras. Com sucesso, essas empresas vêm se utilizando das categorias designadas de Socialização, Externalização, Combinação e Inter- 
nalização, ou modelo SECl, elaboradas pelos autores para estabelecer complexos sistemas de gestão da informação e de conhecimento com base na divulgação do conhecimento. "O conhecimento pode ser definido como uma capacidade para ação"; essa é uma derivação do famoso jargão "conhecimento é poder", de Francis Bacon, que sugere que o "[...] conhecimento deriva sua utilidade da capacidade de pôr alguma coisa em movimento" (Stehr, 2008, p.223). Entretanto, na maioria dos casos, essa afirmação é apropriada pelo modus operandi e pela cultura organizacional, constituindo-se uma barreira às ações de compartilhamento do conhecimento. Entendemos o poder que o conhecimento tem, principalmente, nas ações que os sujeitos podem desenvolver a partir dele, pois ele possibilita liberdade, e, no âmbito do desenvolvimento de uma ação, potencializa a reflexão sobre a prática: a práxis.

Segundo Moresi e Mendes (2010), o compartilhamento de conhecimento tem como prerrogativa o direcionamento do fluxo de conhecimento para um ponto central, obedecendo à dinâmica da coleta, da análise e da avaliação. Concorda-se com essas fases, mas compreendidas como uma primeira etapa desse processo. Para que se tenha uma coerência cíclica e o caráter social do compartilhamento, a disseminação do conhecimento coletado precisa ser a etapa seguinte, após a avaliação.

No âmbito institucional, a Disseminação Seletiva de Informações (DSI) pode ser utilizada como um método, sendo muito apropriada a definição citada por Souto (2010, p.9), que diz ser um "[...] serviço dentro de uma organização que se refere à canalização de novos itens de informação, vindos de quaisquer fontes para aqueles pontos dentro da organização onde a probabilidade de utilização, em conexão com atividades ou interesses, é alta". É exatamente o que o Núcleo pretende, ou seja, coletar estoques de informações e conhecimentos em propriedade de poucos, e disseminá-los para que os demais interessados, setores que desempenham atividades afins, tenham acesso a eles e possam compartilhá-los. Esse frescor conferido ao movimento do conhecimento na instituição, a partir da adição da DSI, expõe uma característica social da gestão da informação por considerar que o compartilhamento do conhecimento aparece em dois momentos: 1) quando da colaboração para a coleta daqueles que detêm o do conhecimento tácito; e 2) quando compartilhado com os interessados após estar explícito.

Desse modo, o projeto do Núcleo de Gestão da Informação e Inteligência da ESDEP tem como fundamentação as categorias de compreensão da espiral do conhecimento de Nonaka eTakeuchi (1995), entendendo que a criação do conhecimento é um processo contínuo de interações dinâmicas entre o tácito e o explícito. Embora se trate de um projeto que visa à gestão da informação, a espiral do conhecimento de Nonaka e Takeuchi apoia o entendimento de procedimento contínuo no fluxo informacional no Núcleo da ESDEP (Figura 1).

Na socialização, acontece o compartilhamento do conhecimento tácito, sendo a experiência compartilhada; na externalização, os conceitos são identificados e combinados; na combinação, as informações são organizadas; e, na internalização, é a possibilidade do momento de catarse, em que o"outro" pode desenvolver novos conhecimentos ao ter acesso a essas informações (Nonaka; Takeuchi, 1995). Compreende-se que essas quatro modalidades da conversão do conhecimento interagem em forma de espiral, sendo relevante para introduzir na reali-

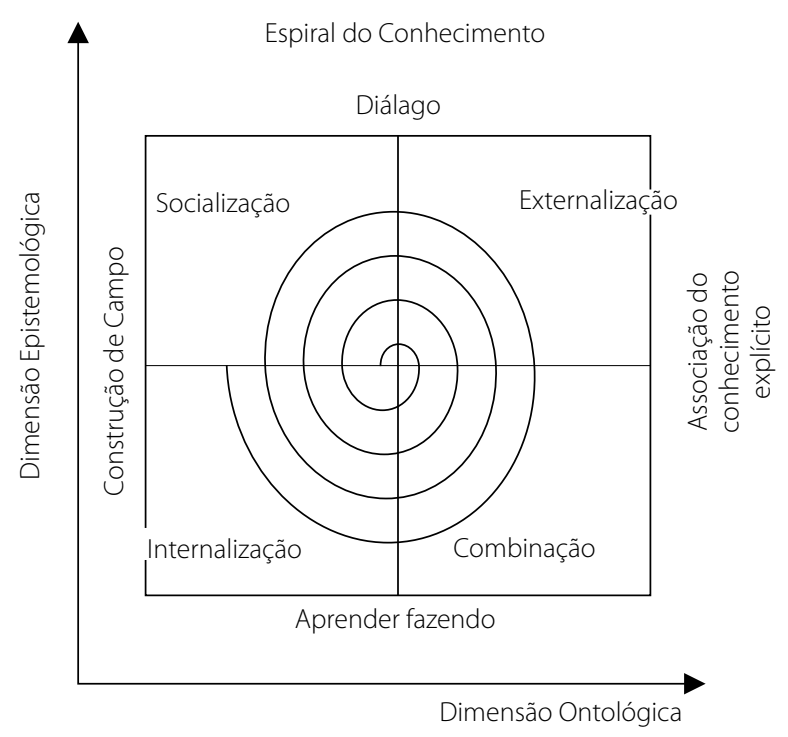

Figura 1. SECI.

Fonte: Nonaka e Takeuchi (1995). 
dade da Defensoria Pública o entendimento que a socialização da informação, externalizada, estruturada e organizada contribui como mais uma variável da missão educadora da ESDEP, aumentando gradativamente o conhecimento da instituição DPE.

A gestão da informação, embora condicione diretamente em seus objetivos e conceitos o direcionamento às instâncias intelectuais de pessoas e organizações, também pode ser compreendida como uma maneira de contribuir com a inclusão. Nessa perspectiva, entende-se que a gestão da informação, se utilizada para compartilhar, divulgar e disseminar informações, que até então eram de conhecimento de poucos, proporcione a inclusão de maiores parcelas do público, criando um coletivo.

O acesso à informação sempre esteve ligado à inclusão social, como pontuam Burke e Ornstein (1998), relatando que os ancestrais do homem moderno usaram, desde os primeiros assentamentos agrícolas, passando pela criação dos numerais e do alfabeto, o conhecimento sobre técnicas já dominadas, visando manter, reforçar e centralizar o domínio sobre a sociedade, beneficiando a um pequeno grupo. Durante todo este tempo:

[...] cresceu sem cessar o abismo entre os poucos detentores do saber esotérico que conferia poderes de corte-e-controle sobre a sociedade e a maioria dos que não o compreendiam. E mesmo a fabricação de instrumentos tendo gerado, do bastão do xamã ao alfabeto, uma quantidade de conhecimento sempre maior e mais acessível, devemos nos lembrar jamais este acesso esteve disponível para mais do que uma fração minúscula da população (Burke; Ornstein, 1998, p.77).

As informações e os conhecimentos restritos a poucas pessoas terminam por alimentar as diferenças, seja no âmbito da sociedade como no âmbito organizacional, criando um abismo. Assim, considerou-se rele- vante contemplar a proposta da gestão da informação neste projeto, visando a disseminar para todos os membros da instituição e da comunidade as informações adquiridas por aqueles que tiveram acesso aos cursos, workshops etc., disponibilizados pela Instituição. Vale pontuar com referência a este projeto a abordagem dada a sua metodologia de concepção. O viés da inclusão social também foi contemplado com a proposta de se treinarem os adolescentes "menores aprendizes" em cumprimento de medida socioeducativa, oriundos da Fundação Cidade Mãe, para desenvolver a coleta das informações com os membros beneficiados.

\section{Métodos}

Embora se trate da descrição da implementação de um projeto, faz-se interessante circunscrever o traçado metodológico que todo experimento sustenta: trata-se de um estudo de caso, que prestigia a observação direta, com métodos de coleta de dados baseados em formulário de questões norteadoras e roteiro de entrevista semiestruturado. Os participantes principais (coletores e multiplicadores da informação) são os adolescentes em cumprimento de medida socioeducativa, na condição de aprendizes do projeto. Os demais participantes são os defensores e funcionários contemplados com algum tipo de investimento, voltado para qualificação profissional, viabilizado pela ESDEP.

O Núcleo de Gestão da Informação e Inteligência da ESDEP visa a atender um público-alvo formado pelos defensores da sua sede em Salvador e das cinco regionais - Feira de Santana, Itabuna, Ilhéus, Vitória da Conquista, Santo Antônio de Jesus - que atendem a 34 comarcas.

Desse modo, o público real do Núcleo compreende os defensores públicos e os servidores, e o público

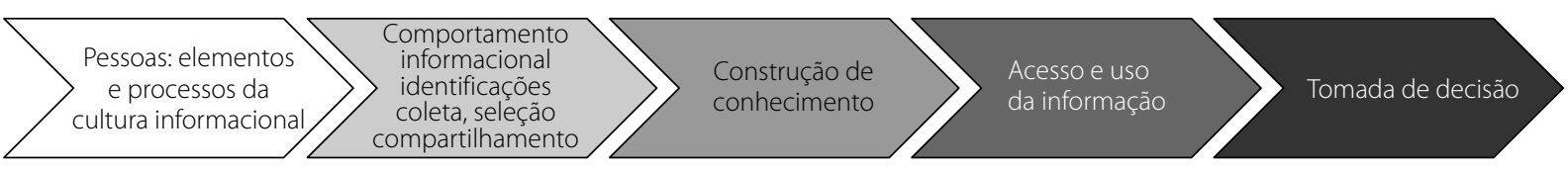

Figura 2. Fluxo proposto de Gestão da Informação.

Fonte: Elaboração própria com base em Nonaka e Takeuchi (1995) e Souto (2010).

TransInformação, Campinas, 24(1):39-46, jan./abr., 2012 
potencial compreende os estagiários de nível superior, os estagiários de nível médio e os interessados em seu acervo.

Destacam-se dois tipos de conhecimento na organização: o explícito: transmitido por meio de linguagem formal; o tácito: transmitido através de exemplos e da convivência (Figura 2).

Para alcançar os objetivos deste projeto foi necessário seguir alguns passos com base em uma metodologia de trabalho, procurando seguir a linha de Nonaka e Takeuchi (1995).

- Passo 1: preparar formulários de coleta de informação; treinar os menores aprendizes para o processo de coleta.

- Passo 2: a) Processos de transmissão do conhecimento: combinação (explícito para explícito); internalização (explícito para tácito); socialização (tácito para tácito); externalização (tácito para explícito). b) Processo de coleta: gravação da informação/conhecimento. c) Processamento técnico - tombamento; classificação, catalogação: etiquetagem eletromagnética e código de barra do sistema; processamento técnico de cada unidade de informação antes de disponibilizar o conteúdo ao público. d) Resultado esperado - compartilhamento e inclusão: disseminação Seletiva da Informação - potencial da globalização do conhecimento, aja vista que não somente o contemplado com a qualificação profissional terá a possibilidade utilizar em sua atividade, mas todos aqueles do setor interessado; inclusão social - oferecer mais uma atividade aos adolescentes em cumprimento de medida, visando socioeducação, contato com as matérias defensoriais, resultando em apropriação da Instituição, além de dar-Ihes oportunidade de desempenhar na prática atividades instrumentais da área da Biblioteconomia.

Moresi e Mendes (2010) apontam que um dos fatores que influenciam o compartilhamento da informação e do conhecimento é o apoio prático aos processos de compartilhamento. Assim, identificou-se que há vontade e interesse em compartilhar por parte daqueles que tiveram acesso às informações através dos eixos de qualificação externa da ESDEP, além de condições, tecnologias, pessoal interessado e orientações técnicas, para que o compartilhamento aconteça.

\section{Considerações Finais}

A gestão da informação vem apresentando excelentes resultados em grandes organizações, que pretendem universalizar as informações e com isso manter um ambiente informacionalmente integrado.

De maneira direta e objetiva, visa-se com o Núcleo de Gestão da Informação e Inteligência da Escola Superior da Defensoria Pública proporcionar conhecimento tácito (dos membros da DPE - que participam de eventos) em explícito, colaborando com a inteligência organizacional da Defensoria Pública do Estado. Para tanto, coletam-se as informações dos membros e servidores que participam dos eventos viabilizados pela ESDEP; armazena-se o conteúdo em mídias alocadas na Biblioteca da ESDEP, possibilitando o acesso daqueles que não participaram da atividade (evento, congresso etc.); gerencia-se a informação de modo a sistematizá-la e torná-la disponível ao público de usuários da Biblioteca da ESDEP para posterior disseminação do conhecimento (antes tácito), agora de forma explícita, proporcionando socialização e fortalecimento da missão da ESDEP.

Para concluir, cumpre assinalar que esta comunicação tem o intuito de explicitar a proposta do Núcleo e alguns aspectos de sua fundamentação. Vale ainda salientar que não se teve a intenção de exaurir toda a contextualização que norteou este projeto, que foi aprovado em âmbito estadual e agraciado com o prêmio Ideias e Ações Inovadoras do Estado.

\section{Referências}

BORGES, M.E.N.; SOUZA, M.C.V. Serviços e produtos de informação para empresas: um desafio estratégico para os profissionais de informação. In: PAIM, I. (Org.). Gestão da informação e do conhecimento. Belo Horizonte: UFMG, 2003.
BURKE, P.; ORNTEIN, R. O presente do fazedor de machados: os dois gumes da história. Rio de Janeiro: Bertrand, 1998.

MOLINA, L.G. Gestão da informação e do conhecimento e as TICs aplicadas aos portais corporativos. In: VALENTIM, M. 
Gestão da informação e do conhecimento. São Paulo: Polis, 2008.

MORESI, E.A.D.; MENDES, S.P. Compartilhamento do conhecimento em portais corporativos. TransInformação, v.22, n.1, p.19-32, 2010.

NONAKA, I.; TAKEUCHI, H. The knowledge-creating company. New York: Oxford University Press, 1995.

OLIVEIRA, M.; BERTUCCI, M.G.E.S. As pequenas e médias empresas e a gestão da informação. In: PAIM, I. (Org.). Gestão da informação e do conhecimento. Belo Horizonte: UFMG, 2003.

SILVA, A.M.; RIBEIRO, F. Das ciências documentais à ciência da informação: ensaio epistemológico para um novo modelo curricular. Porto: Afrontamento, 2008.
SOUTO, L.F. Informação seletiva, mediação e tecnologia: a evolução dos serviços de disseminação seletiva da informação. Rio de Janeiro: Interciência, 2010.

STEHR, N. Liberdade é filha do conhecimento? Tempo Social, v.20, n.2, p.221-234, 2008.

STEWART, T.A. Intellectual capital. Fortune Magazine, 3 june 1991. Available from: <http://money.cnn.com/ magazines/fortune/fortune_archive/1991/06/03/75096/ index.htm>.

VALENTIM, M.L.P. Inteligência competitiva em organizações: dado, informação e conhecimento. DataGramaZero, v.3, n.4, 2002. Disponível em: <www.dgz.org.br>. 